Centre de recherche en neurobiologie-

neurophysiologie de Marseille, CRN2M,

département de physiologie neurovégétative,

UMR 6231 CNRS, USC INRA 2027,

La mPGES- 1 :

elle nous rend malades!

Université Paul Cézanne

et Université de la Méditerranée,

Faculté des sciences et techniques de Saint-Jérôme,

avenue Escadrille Normandie-Niemen,

Émilie Pecchi, Michel Dallaporta, Sylvie Thirion,

André Jean, Jean Denis Troadec

13397 Marseille Cedex, France.

emilie.pecchi@univ-cezanne.fr

j-d.troadec@univ-cezanne.fr

L'infection, réaction

de phase aiguë et illness behavior

Une infection par un pathogène induit au sein

de l'organisme de profonds changements physiologiques et comportementaux, dont l'ensemble est connu sous le terme de «réaction de phase aiguë» (acute phase reaction). Subdivisée en composantes locale, systémique et centrale, la réaction de phase aiguë est le résultat de l'activation des cellules du système immunitaire inné qui reconnaissent les

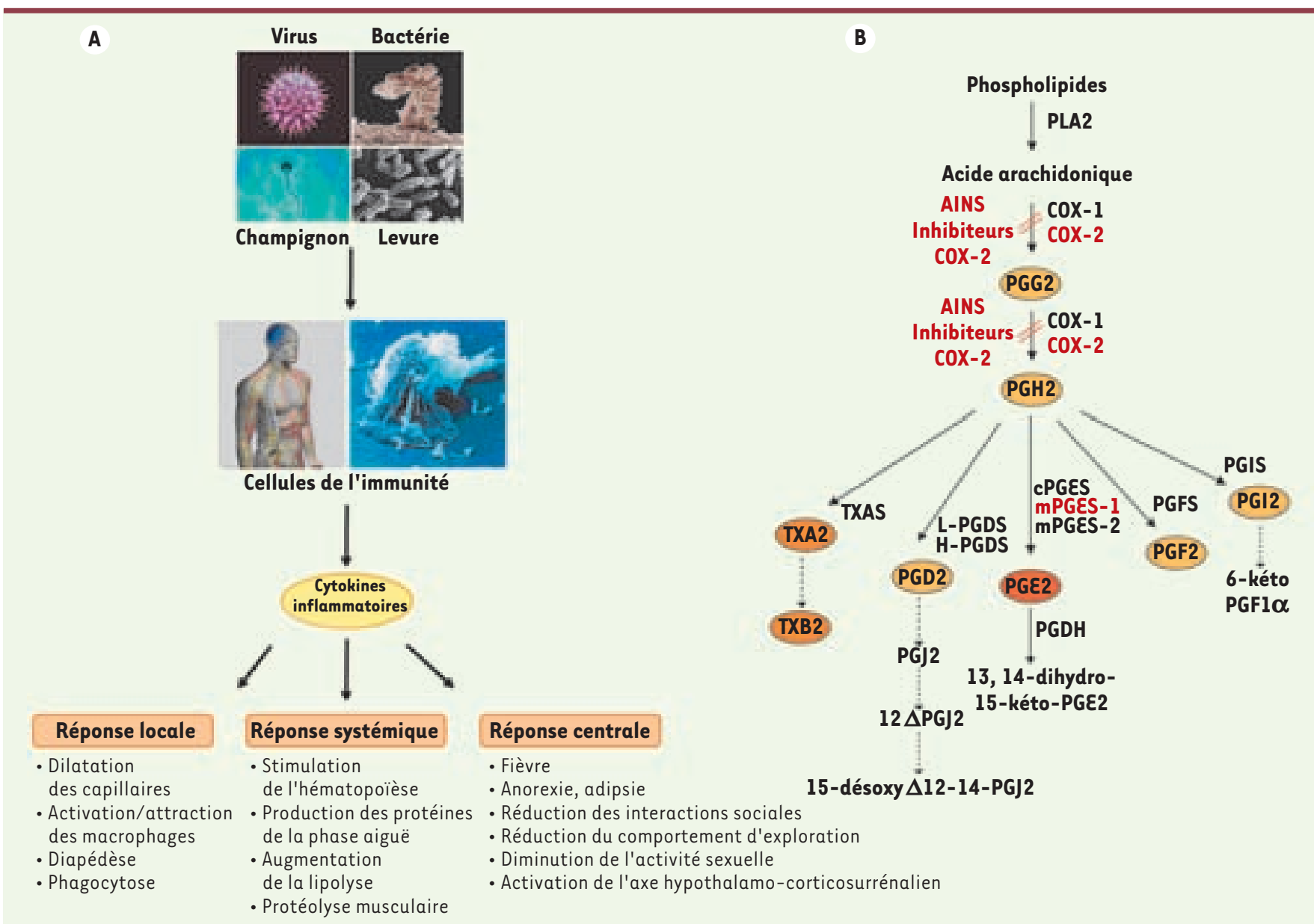

Figure 1. Les composantes de la réaction de phase aiguë et la biosynthèse des prostanoïdes. $A$. Lors d'une infection, les cellules du système immunitaire inné détectent la présence de pathogènes au sein de l'organisme. Leur activation induit la libération de cytokines inflammatoires responsables de la mise en place des différentes composantes de la réaction de phase aiguë, c'est-à-dire locale, systémique et centrale. B. L'acide arachidonique est libéré à partir des phospholipides membranaires par les phospholipases A2 (PLA2). II subit alors une réaction d'oxygénation permettant la formation de PGG2 puis une réaction de peroxydation permettant la formation de PGH2. Ces deux réactions sont assurées par les cyclooxygénases (COX). La PGH2 ainsi formée subit une isomérisation dépendante de l'enzyme terminale. Les TXAS, PGDS, PGES, PGIS et PGFS catalysent respectivement la formation de la TXA2, PGD2, PGE2, PGI2 et PGF2. AINS : anti-inflammatoires non stéroïdiens; PG : prostaglandine ; TX : thromboxane ; PGDS : PGD synthase ; PGES : PGE synthase ; PGFS : PGF synthase ; PGIS : PGI synthase ; TXAS : TXA synthase. 
motifs moléculaires associés aux pathogènes. La composante locale est un processus dynamique, immédiat et transitoire. Elle se traduit cliniquement par les signes classiques de l'inflammation aiguë: rougeur, chaleur, tuméfaction et douleur. Ces effets résultent de la vasodilatation locale des capillaires sanguins, de l'augmentation de leur perméabilité aux protéines plasmatiques, de l'attraction, l'activation et la diapédèse des macrophages au niveau du foyer inflammatoire, et de la phagocytose des particules immunogènes et des débris cellulaires. En cas d'infection sévère, cette réaction inflammatoire peut s'étendre à l'ensemble de l'organisme, entraînant des modifications systémiques avec une activation de l'hématopoïèse, une augmentation de la sécrétion hépatique des protéines de phase aiguë, une augmentation de la lipolyse et de la protéolyse musculaire. Toutes ces modifications sont coûteuses en énergie et demandent à l'organisme de redéfinir ses priorités physiologiques afin de s'adapter efficacement à ces contraintes. Cette adaptation nécessite en particulier l'intervention du système nerveux central, qui coordonne la mise en place de la composante centrale de la réaction de phase aiguë. Outre la fièvre et l'activation de l'axe hypothalamo-corticosurrénalien, elle comprend également de profonds changements comportementaux regroupés sous le terme de «comportement lié à la maladie» (illness behavior) [1]. Les individus malades sont asthéniques, apathiques et incapables de se concentrer. De plus, ils montrent peu d'intérêt vis-à-vis de leur environnement et réduisent leur prise hydrique et alimentaire (anorexie inflammatoire).

\section{Inhibition spécifique}

\section{de l'activité COX-2 : espoirs déçus}

La mise en place du illness behavior repose sur la modification de l'activité de structures cérébrales, responsables par exemple du maintien de la température corporelle ou de

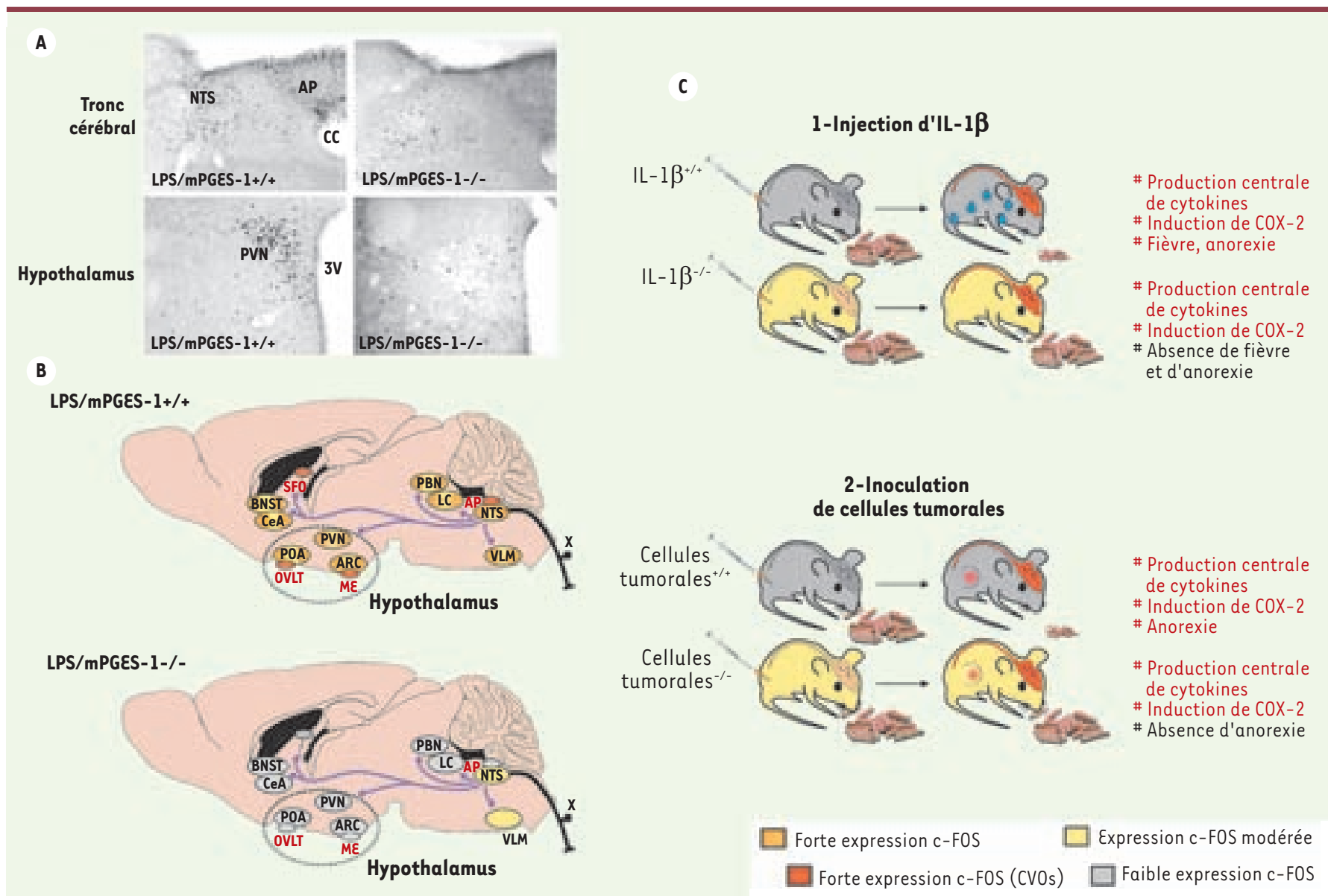

Figure 2. Réponse des souris invalidées pour la mPGES-1 aux signaux inflammatoires. A-B. Visualisation de l'activation des structures centrales en réponse à l'injection de lipopolysaccharide à l'aide du marqueur c-Fos. AP : area postrema ; POA : aire pré-optique; ARC: noyau arqué de l'hypothalamus; BNST : lit de la strie terminale ; CeA : amygdale centrale ; ME : éminence médiane ; LC : locus cœruleus ; mPGES : prostaglandine $\varepsilon$ synthase microsomale ; NTS : noyau du tractus solitaire ; CVO : organe circumventriculaire; OVLT : organe vasculaire de la lame terminale; PBN : noyau parabrachial ; PVN : noyau paraventriculaire de l'hypothalamus ; SFO : organe subfornical; VLM : medulla ventro-latérale; $X$ : nerf vague. C. L'invalidation de la mPGES-1 prévient l'apparition de la fièvre et de l'anorexie lors d'une inflammation aiguë induite par l'injection d'IL-1 $\beta$. Les souris $m P G E S-I^{-/-}$(jaune) sont également résistantes à l'anorexie normalement observée lors d'une inflammation chronique résultant de la croissance de certaines tumeurs. NB : le tas de nourriture traduit la quantité de nourriture consommée et non la nourriture restante. 
I'homéostasie énergétique, par des cytokines inflammatoires produites en périphérie en réponse à une infection ou une inflammation. Ainsi, au niveau central, la synthèse de la prostaglandine de type દ2 (PGE2) à partir de l'acide arachidonique augmente de façon considérable lors d'une infection et son rôle causal dans la mise en place des symptômes du illness behavior a été établi voilà plusieurs années par différents groupes $[2,3,13]$. Une des stratégies thérapeutiques anti-inflammatoires parmi les plus anciennes consiste à bloquer la production de ces composés via l'inhibition des enzymes cyclo-oxygénases (COX) dont deux principales isoformes sont actuellement identifiées: COX-1 et COX-2. Tandis que COX-1, une enzyme constitutive, participe à la production de PG\&2 nécessaire à I'homéostasie tissulaire, COX-2, dont l'expression est fortement stimulée par l'inflammation, est responsable de la synthèse de PGE2 en condition inflammatoire.

Les propriétés anti-inflammatoires et antipyrétiques des anti-inflammatoires non stéroïdiens (AINS) reposent en grande partie sur leur capacité à prévenir la production de prostaglandines en ciblant les enzymes COX. Mais leur faible sélectivité COX-2 versus COX-1 explique les effets secondaires de ces AINS. En inhibant la production de PG\&2 dépendante de COX-1, ces composés perturbent l'homéostasie de certains tissus, dont la muqueuse gastro-intestinale, entraînant par exemple des troubles digestifs [4]. Pour limiter ces effets délétères, un effort a été entrepris au début des années 1990, afin de développer des inhibiteurs ciblant plus spécifiquement COX-2. Malheureusement leur utilisation n'est pas dépourvue d'inconvénients. En effet, bien qu'ils n'entraînent plus de perturbations majeures de la sphère gastro-intestinale, ils sont associés à un risque accru d'hypertension, d'infarctus du myocarde et d'accident vasculaire cérébral. Ces désordres cardiovasculaires semblent être soustendus par une altération de la balance entre deux prostanoïdes : la prostaglandine 12 aux propriétés anti-agrégante et vasodilatatrice et la thromboxane A2 possédant des actions opposées [5].

\section{La mPGES-1 : actrice essentielle} du « comportement lié à la maladie » L'inhibition des enzymes COX étant invariablement associée à des effets secondaires, l'attention s'est alors focalisée sur les enzymes intervenant en aval des COX et catalysant la production d'un type spécifique de prostanoïdes. L'activité COX permet en effet la formation de $\mathrm{PGH2}$, précurseur commun à tous les prostanoïdes. La PGH2 subit ensuite une isomérisation dépendante de l'enzyme terminale. Dans le cas de la PGE2, ce sont les prostaglandine $\varepsilon$ synthases (PGES) qui permettent l'isomérisation de la PGH2 en PGE2. Alors que l'activité PGES est décrite depuis les années 1970, l'identification des enzymes est relativement récente [6]. Actuellement les gènes de trois isoformes des PGES ont été clonés: ceux de la PGES cytosolique (cPGES) et des PGES microsomales de type 1 et 2 (mPGES- 1 et -2 ). La cPGES et la mPGES-2 sont exprimées constitutivement et sont responsables de la synthèse de PGE2 nécessaire à I'homéostasie tissulaire en coordination avec COX-1. En revanche, la mPG\&S-l est induite lors de stimulus inflammatoires et son expression est alors étroitement corrélée à l'expression de COX-2 et à la synthèse de PGE2. La caractérisation du rôle spécifique de ces enzymes PGES et plus particulièrement de celui de la mPGES-l dans la mise en place des symptômes consécutifs à l'inflammation a considérablement progressé ces dernières années, au point que l'inhibition de la mPGES-1 apparaît aujourd'hui comme une stratégie anti-inflammatoire prometteuse.

L'absence d'inhibiteurs spécifiques de cette enzyme explique les difficultés de l'analyse du rôle de la mPGES-l dans la symptomatologie liée à l'inflammation. Ce problème méthodologique fut contourné grâce à la création de souris transgéniques dont le gène codant pour la mPGES-1 (Ptges) a été invalidé [7]. En utilisant ces souris Ptges ${ }^{-/}$, Engblom et ses collaborateurs ont montré que l'absence de cette enzyme suffit à prévenir la réponse fébrile normalement observée en réponse à l'injection de lipopolysaccharides bactériens [8]. La synthèse de PGE2 induite en réponse au LPS dans I'hypothalamus de ces animaux Ptges $^{-/-}$est fortement atténuée par rapport à celle d'animaux sauvages. En accord avec ces résultats, une autre équipe démontra que les souris invalidées pour la mPGES-l étaient également résistantes à la réponse fébrile induite par l'injection d'une cytokine inflammatoire, l'interleukine-1 $\beta$ (IL-1 $\beta$ ) [9]. Parallèlement, notre laboratoire a démontré le rôle essentiel de cette enzyme dans l'anorexie observée lors d'une inflammation aiguë induite par une injection d'IL-1 $\beta$ [10]. De plus, nous avons également pu montrer l'implication de la mPGES-1 dans le syndrome anorexie/cachexie (inflammation chronique) qui se développe en réponse à l'implantation sous-cutanée d'une tumeur sécrétrice de cytokines [11]. Dans ces deux modèles, l'invalidation de la mPGES-1 abolit la réponse anorexique des animaux et limite ainsi leur perte de poids $[10,11]$. Il est important de noter que cet effet sur l'appétit intervient alors que l'expression centrale de COX-2 est augmentée chez ces animaux et que l'expression constitutive des enzymes mPGES-2 et CPGES n'est pas modifiée [10,11]. En accord avec ces résultats comportementaux, l'activation des structures cérébrales responsables du maintien de la température corporelle et de l'homéostasie énergétique en réponse à une inflammation périphérique est très diminuée chez les souris invalidées pour la mPGES-1 [12].

\section{Conclusion}

Au total, l'ensemble de ces données indique que l'inhibition de la mPG\&S-1 peut constituer une stratégie thérapeutique 
innovante permettant de limiter l'apparition des symptômes non spécifiques associés à la maladie comme la fièvre et l'anorexie. En limitant spécifiquement la synthèse de la PGE2 en condition inflammatoire, tout en conservant la synthèse des autres prostanoïdes dérivant de l'activité COX, des inhibiteurs spécifiques de la mPGES-l devraient pouvoir exercer un effet anti-inflammatoire ciblé, dépourvu des effets secondaires rencontrés actuellement avec les AINS et les inhibiteurs spécifiques de COX-2. $\diamond$ mPGES-1: she makes us sick!

\section{RéFÉRENCES}

1. Hart BL. Biological basis of the behavior of sick animals. Neurosci Biobehav Rev 1988; 12: 123-37.
2. Johnson PM, Vogt SK, Burney MW, Muglia LJ. COX-2 inhibition attenuates anorexia during systemic inflammation without impairing cytokine production. Am J Physiol Endocrinol Metab 2002 ; 282 : ع650-6.

3. Lugarini F, Hrupka BJ, Schwartz GJ, et al. A role for cyclooxygenase-2 in lipopolysaccharide-induced anorexia in rats. Am J Physiol Regul Integr Comp Physiol 2002; 283 : R862-8.

4. McCarthy D. Nonsteroidal anti-inflammatory drugrelated gastrointestinal toxicity: definitions and epidemiology. Am J Med 1998 ; 105 : 3S-9S.

5. Fries $S$, Grosser T. The cardiovascular pharmacology of COX-2 Inhibition. Am Soc Hematol Educ Program $2005 ; 445-51$.

6. Jakobsson PJ, Thoren S, Morgenstern R, Samuelsson B. Identification of human prostaglandin $\varepsilon$ synthase: a microsomal, glutathione-dependent, inducible enzyme, constituting a potential novel drug target. Proc Natl Acad Sci USA 1999; $96: 7220-5$

7. Trebino C $\varepsilon$, Stock JL, Gibbons CP, et al. Impaired inflammatory and pain responses in mice lacking an inducible prostaglandin $\varepsilon$ synthase. Proc Natl Acad Sci USA 2003; 100 : 9044-9.

8. Engblom D. Saha S. Engstrom L, et al. Microsomal prostaglandin $\varepsilon$ synthase- 1 is the central switch during immune-induced pyresis. Nat Neurosci 2003; $6: 1137-8$.

\section{NOUVELLE}

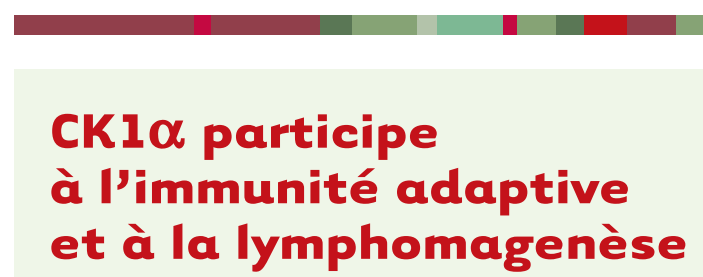

Nicolas Bidère

$>$ Le facteur de transcription NF- $\kappa B$ joue un rôle vital dans le développement, la survie et le maintien de l'homéostasie du système immunitaire [1]. Présent de manière constitutive dans le cytosol, l'hétérodimère $N F-\kappa B$ (le plus souvent p65/p50) est séquestré par son inhibiteur $1 \kappa B \alpha$ qui masque sa séquence de localisation nucléaire $[2,3]$. L'activation de $N F-\kappa B$ repose sur la mise en place de plates-formes multiprotéiques spécifiques d'un stimulus dont la fonction est de recruter et rendre compétent le complexe IKK (inhibitor of NF-KB kinase). IKK phosphoryle alors $I \kappa B \alpha$, ce qui permet sa dégradation par le protéasome. Libéré $d^{\prime} \mid \kappa B \alpha, N F-\kappa B$ migre vers le noyau où il déclenche la transcription de ses gènes cibles (Figure 1).

\section{CARMA 1 et le complexe CBM}

Dans les lymphocytes, la stimulation antigénique assemble un « signalosome »dont la composition demeure mal caractérisée [4]. En particulier, la protéine d'échafaudage CARMAl (caspase recruitment domain-containing membrane-associated guanylate kinase protein-1), et l'hétérodimère $\mathrm{BCL} 10$ / MALTl (mucosa-associated lymphoid tissue) (complexe CBM) émergent comme des acteurs essentiels de la machinerie $\mathrm{NF}-\kappa \mathrm{B}$ (Figure 1A) [5-7]. La protéine kinase $C$ (PKC) $\theta$ ou $\beta$, dans les cellules $T$ et $B$, respectivement, recrute et phosphoryle CARMAl dans les microdomaines des radeaux lipidiques. CARMAl change alors de conformation et autorise l'association de BCL10/MALTI nécessaire à la formation du signalosome [8]. Le CBM
9. Saha S, Engstrom L, Mackerlova L, et al. Impaired febrile responses to immune challenge in mice deficient in microsomal prostaglandin $\varepsilon$ synthase-1. Am J Physiol Regul Integr Comp Physiol 2005; 288: R1100-7.

10. Pecchi $\varepsilon$, Dallaporta $M$, Thirion S, et al. Involvement of central microsomal prostaglandin $\varepsilon$ synthase- 1 in IL-lbeta-induced anorexia. Physiol Genomics 2006; $25:$ 485-92.

11. Pecchi $\varepsilon$, Dallaporta M, Jean A, et al. mPGES-1 knock-out mice are resistant to cancer-induced anorexia despite the absence of central mPGES-1 upregulation in wild-type anorexic mice. J Neuroimmunol 2008; 199 : 104-14.

12. Dallaporta M, Pecchi $\varepsilon$, Jacques C, et al. c-Fos immunoreactivity induced by intraperitoneal LPS administration is reduced in the brain of mice lacking the microsomal prostaglandin $\varepsilon$ synthase-1 (mPGES-1). Brain Behav Immun 2007 ; 21 : 1109-21.

13. Malki S, Declosmenil F, Farhat A, et al. La prostaglandine D2 : nouveaux rôles dans la gonade embryonnaire et pathologique. Med Sci (Paris) 2008 ; $24: 177-83$.
Inserm U542, Université Paris-Sud, Hôpital Paul Brousse, 94800 Villejuif, France. nicolas.bidere@inserm.fr

participe également à la propagation des lymphomes $B$ de type $A B C-D L B C L$ (activated $B$ cell-like diffuse large $B$-cell lymphoma), dont la survie repose sur une activité aberrante de NF-KB [9]. Comprendre les modes de régulation du CBM en conditions physiologiques comme pathologiques se révèle donc être un enjeu majeur.

Le double jeu de la caséine kinase $1 \alpha$ Afin de caractériser de nouveaux régulateurs du CBM, deux cribles furent menés parallèlement: une recherche protéomique par spectrométrie de masse de partenaires de CARMAl, et le criblage génétique d'une banque de 1854 vecteurs shARN ciblant 683 gènes afin d'identifier lesquels parmi ceux-ci sont requis pour la croissance des ABC-DLBCL. Dans les 